\title{
A Prospective Study of Posttraumatic Stress Disorder Symptoms and Coronary Heart Disease in Women
}

\author{
Laura D. Kubzansky, \\ Department of Society, Human Development, and Health, Harvard School of Public Health \\ Karestan C. Koenen, \\ Department of Society, Human Development, and Health, Harvard School of Public Health \\ Cynthia Jones, and \\ Department of Mental Health, Johns Hopkins Bloomberg School of Public Health \\ William W. Eaton \\ Department of Mental Health, Johns Hopkins Bloomberg School of Public Health
}

\begin{abstract}
Objective-Posttraumatic stress disorder (PTSD) reflects a prolonged stress reaction and dysregulation of the stress response system and is hypothesized to increase risk of developing coronary heart disease (CHD). No study has tested this hypothesis in women even though PTSD is more prevalent among women than men. This study aims to examine whether higher levels of PTSD symptoms are associated with increased risk of incident CHD among women.
\end{abstract}

Design-A prospective study using data from women participating in the Baltimore cohort of the Epidemiologic Catchment Area study $(n=1059)$. Past year trauma and associated PTSD symptoms were assessed using the NIMH Diagnostic Interview Schedule.

Main Outcome Measures-Incident CHD occurring during the 14-year follow-up through 1996.

Results-Women with five or more symptoms were at over three times the risk of incident CHD compared with those with no symptoms (age-adjusted OR $=3.21,95 \%$ CI: $1.29-7.98$ ). Findings were maintained after controlling for standard coronary risk factors as well as depression or trait anxiety.

Conclusion-PTSD symptoms may have damaging effects on physical health for civilian community-dwelling women, with high levels of PTSD symptoms associated with increased risk of CHD-related morbidity and mortality.

\section{Keywords}

coronary disease; posttraumatic stress disorder; depression; stress; women

Posttraumatic stress disorder (PTSD) reflects a prolonged stress reaction and dysregulation of the stress response system (Vanitallie, 2002). Evidence that dysregulated stress responses are associated with atherosclerosis and other cardiovascular system damage has suggested that PTSD may increase risk of developing coronary heart disease (CHD) (Rozanski, Blumenthal, Davidson, Saab, \& Kubzansky, 2005). In a recent prospective study among older men who

(C) 2009 American Psychological Association

Correspondence concerning this article should be addressed to L. Kubzansky, Department of Society, Human Development, and Health, Harvard School of Public Health, 677 Huntington Avenue, Boston, MA 02115. lkubzans@hsph.harvard.edu. 
served in the military, risk of incident CHD was greater among men with higher versus lower levels of PTSD symptoms (Kubzansky, Koenen, Spiro, Vokonas, \& Sparrow, 2007). Although PTSD is highly prevalent among war veterans (Hoge, Castro, Messer, McGurk, Cotting, \& Koffman, 2004), it is also common in the general population and in fact is more prevalent among women (Kessler, Berglund, Demler, Jin, Merikangas, \& Walters, 2005). Women's risk of developing PTSD is twice that of men; one in nine women will develop PTSD at some point in their lives (Kessler et al., 2005). Given that CHD is the leading cause of death among older women (Rosamond et al., 2007), the relation of PTSD and CHD incidence in women deserves closer attention. Though health-damaging effects of PTSD are hypothesized to be similar for men and women, no prospective studies have examined PTSD in relation to CHD risk among women or in a nonmilitary population.

PTSD is defined by the combination of exposure to a traumatic event and the occurrence of three types of symptoms: reexperiencing the traumatic event, avoidance of traumatic reminders and emotional numbing, and hyper-arousal. Though much of the work on PTSD and cardiovascular risk has been conducted among male combat veterans, there is little evidence to suggest that potential health-damaging effects of PTSD are constrained to this population. In the limited existing work that considers both men and women civilians with PTSD, a higher prevalence of cardiovascular disease and related risk factors has been documented (Spindler $\&$ Pedersen, 2005). Moreover, consistent with other work suggesting that PTSD rather than trauma exposure alone may mediate between trauma and risk of adverse health outcomes (Kang, Bullman, \& Taylor, 2006), Dong and colleagues found that civilian men and women with numerous adverse childhood experiences were at greater risk of ischemic heart disease [Odds Ratio $(\mathrm{OR})=3.6,95 \%$ Confidence Interval $(\mathrm{CI})\}=2.4-5.3]$, an effect that was explained more completely by psychological distress than by traditional risk factors (Dong et al., 2004).

The present study provides a prospective test of the hypothesis that PTSD symptoms are associated with increased risk of developing CHD among community dwelling women. Data are from the Baltimore cohort of the Epidemiologic Catchment Area (ECA) study, a survey of psychiatric disorders in the general population. Work in this and other samples has considered the relationship between depression or anxiety and CHD (Kubzansky \& Kawachi, 2000; Pratt, Ford, Crum, Armenian, Gallo, \& Eaton, 1996). To address concerns that an observed association between PTSD and CHD may be because of other forms of distress, this study also considers whether PTSD is related to CHD onset after taking account of depression or anxiety (measured separately from PTSD).

\section{Method}

The Epidemiologic Catchment Area (ECA) program was a national study aimed at measuring the incidence and prevalence of psychiatric disorders in the general population (Regier et al., 1984). Five sites participated in this study between 1980 and 1983 and the final sample included over 20,000 respondents. Participants gave informed consent before the interview. The protocol was approved by the Committee on Human Research of the Johns Hopkins School of Hygiene and Public Health.

The first wave of the Baltimore ECA was conducted in 1980 through 1981 and included a probability sample of 3,481 (62.0\% female) household residents of east Baltimore, a population of 175,211 adults. The completion rate for the first wave survey was $82 \%$. The second wave of the Baltimore ECA study ( $n=2768$ participants, $62.9 \%$ female), conducted in 1982, included an assessment of PTSD and therefore serves as the baseline for the current study. The Baltimore ECA Follow-up study involved a third wave of interviews, conducted in 1993 through 1996, and included 1,920 participants (63.1\% female) who completed the first wave interview. Approximately $73 \%$ of those alive were successfully interviewed during this follow-up. Details 
on the study design and methods of the Baltimore ECA follow-up have been described, including work suggesting that PTSD is unlikely to be related to sample attrition (Badawi, Eaton, Myllyluoma, Weimer, \& Gallo, 1999).

Women who completed the second wave Baltimore ECA interview in 1982 and who participated in the subsequent follow-up are included in the present study. Of the 1,741 women who completed the second wave assessment, 354 women (20.3\%) were excluded from the baseline sample because they did not complete the PTSD interview $(n=1)$ had evidence of heart disease in their first or second wave interviews $(n=314)$, or were missing information on health status at baseline $(n=39)$ Of the eligible women 285 were missing either a Wave 3 interview or available death records, and 43 were missing data on heart disease at follow-up, resulting an analytic sample of 1,059 women. No significant differences were evident between women included in versus excluded from the analytic sample with regard to race/ethnicity. However, women excluded from the analyses were significantly more likely to be older, have less than high school education, and have income less than $\$ 14,999$.

\section{Assessment of PTSD}

Past-year PTSD was assessed at the second wave assessment during face-to-face interviews conducted by lay interviewers using the NIMH Diagnostic Interview Schedule (DIS) according to the Diagnostic and Statistical Manual Third Edition (DSM-3) (Robins, Helzer, Croughan, $\&$ Ratcliff, 1981). The PTSD interview began with a gate question to determine whether participants had experienced a traumatic event in the past year. Participants who experienced more than one distressing event were asked to focus on the most distressing event. PTSD symptoms were then queried in relation to that event. Using DSM-3 criteria, only 28 (2.6\%) women in the analytic sample met diagnostic criteria for past-year PTSD. Because of the small number of women who met criteria and related power concerns, PTSD symptom counts were used as a continuous measure for statistical analyses. In addition, to assess the possibility of a threshold effect on CHD risk, symptom levels were categorized into low (0), moderate (1-4) and high (5 or more) based on the distribution of scores in the sample and based on the recognition that diagnosis of PTSD according to the DSM-IV requires five or more symptoms.

\section{Measurement of Other Cardiovascular Risk Factors}

Basic demographic information, including age, race/ethnicity, educational attainment, and income, along with information on history of hypertension or diabetes, smoking status, and alcohol use was collected as part of the second wave $(1,982)$ interviews. Educational attainment was taken from the Wave 1 interview. Family history of heart disease and body mass index (BMI) were assessed at the Wave 3 interview but 234 participants (22\%) were missing information on family history and 263 participants (25\%) were missing information on BMI. Current major depression (yes/no) was assessed at Wave 2 via the DIS according to DSM-3 criteria (Pratt et al., 1996). Trait anxiety was also assessed at Wave 2 with a single item question "Do you now consider yourself a nervous person?" (yes/no).

\section{Assessment of Morbidity and Mortality}

Cardiovascular outcomes were assessed via detailed questions during the third wave interviews (1993-94) as follows. Participants were asked whether they ever had heart trouble. Those who responded affirmatively were then asked to specify the type of heart trouble. The interviewer queried five specific conditions: rheumatic fever, rheumatic heart disease, angina pectoris, a heart attack, and congestive heart failure. Consistent with other work in this area (Dong et al., 2004; Kubzansky \& Koenen, 2007), those who reported having a heart attack or angina pectoris were considered positive for the outcome. Participants were also tracked via the National Death Index and death certificates were obtained for decedents. Death from CHD was designated when a death certificate (coded according to the 9 th revision of the International Classification 
of Diseases) indicated an underlying cause of death coded to rubric 410-414. All other 1993 through 1996 respondents were considered negative for the outcome. Because of the small number of cases for each CHD outcome $(n<30)$, the incident CHD measure combined cases across angina pectoris, nonfatal MI, fatal MI, and other cardiac death.

\section{Data Analysis}

Logistic regression models using the Statistical Analysis System (SAS Institute, 1990) were used to estimate the relative risks of CHD according to level of PTSD, controlling for age (years); race/ethnicity (White/non-White), smoking status (never, former, current); history of high blood pressure (yes/no); history of diabetes (yes/no); whether participants drank two or more drinks of alcohol per day (yes/no), whether participants had completed education beyond high school (yes/no), income ( $<\$ 14,999, \$ 15,000-\$ 24,000,\rangle \$ 25,000)$. Because many participants lacked information on family history of heart disease and body mass index (kg/ $\mathrm{m}^{2}$ ), to maximize our power to detect effects these variables were not included in the primary analyses. Primary analyses consider the association between PTSD symptom level and likelihood of developing CHD. To assess the possibility of threshold effects, analyses subsequently compare the probability of developing CHD across individuals with more versus fewer PTSD symptoms. Odds ratios derived from these analyses represent the effect of PTSD symptom level (low as reference) on CHD risk. Age- and multivariable-adjusted analyses are presented to illustrate the role of potential confounders. Effects of PTSD on noncardiac mortality are also considered. Follow-up analyses examine whether findings change when current depression, trait anxiety, family history, or BMI is included in the models.

\section{Results}

The analytic sample consists of 1,059 women, with mean age of 44.4 years at the start of followup (range $=19-93$ ). Of these women, 59.9\% are White, $48.9 \%$ have high school education or more, and $20.8 \%$ have income $>\$ 25,000$. PTSD symptomatology was skewed toward low levels, with $89.1 \%$ of the sample reporting 0 symptoms, $7.0 \%$ reporting 1 to 4 symptoms, and $4.0 \%$ reporting five or more symptoms. Table 1 demonstrates the association of coronary risk factors measured at baseline with PTSD symptom scores. PTSD symptoms were higher among individuals with less income and among those also reporting depression or trait anxiety. Symptom levels did not differ according to any other covariates.

Of the 1,059 women in the study sample, 86 developed CHD over the follow-up period. There were 30 cases of incident nonfatal MI, 27 cases of fatal MI, 15 cases of other cardiac death, and 14 cases of angina pectoris. Women with more symptoms had greater risk of incident CHD and relative risks for age- and multivariable-adjusted models were virtually identical. For each additional symptom, women were at $17 \%$ increased risk of developing incident CHD (ageadjusted OR $=1.17,95 \%$ CI: $1.06-1.29, p<.01$ ). However, examination of categorical symptom levels suggested a possible threshold effect (see Table 2). Women with five or more symptoms were at approximately three times the risk of developing heart disease compared with those with no symptoms (age-adjusted $\mathrm{OR}=3.21,95 \%$ CI: $1.29-7.98, p<.05$ ). Findings were maintained after controlling for standard coronary risk factors. Women with 1 to 4 symptoms did not appear to be at significantly elevated risk (age-adjusted OR $=1.22$, 95\% CI: $0.46-3.24$, $p=.69$ ).

To ensure that findings could not be attributed to reporting bias (i.e., women with greater distress also report more pain, resulting in more diagnosed angina), the association of PTSD symptoms with fatal and nonfatal MI only was examined. When considering PTSD symptoms as a continuous measure, each additional symptom was associated with $15 \%$ increased risk of incident MI (age-adjusted OR = 1.15, 95\% CI: $1.03-1.29, p<.05$ ). Women with five or more symptoms were at significantly elevated risk for incident MI relative to women with no 
symptoms (age-adjusted OR $=3.25,95 \%$ CI: 1.16-9.11, $p<.05$ ), and effects were similar after adjusting for standard coronary risk factors (see Table 2 ). Sensitivity analyses suggested findings were somewhat attenuated but were maintained when lowering the cut-off for high symptom levels. Trait anxiety and current depression were independently associated with incident CHD in this sample, but controlling for either of these factors did not significantly change any of the associations between PTSD symptoms and incident CHD reported above. For example, when current depression was included in the models the association between PTSD symptoms and total cardiac outcomes was attenuated but still significant (high vs. low PTSD symptom levels multivariable-adjusted OR $=2.91,95 \% \mathrm{CI}: 1.1-7.59, p<.05$ ), and effects of depression remained as well. PTSD symptoms were not significantly associated with all-cause mortality or with mortality after excluding deaths because of CHD (see Table 2).

Another potential concern is whether individuals with PTSD may be more likely to have either family history of heart disease or higher BMI and therefore at greater risk for developing the outcome. Analyses in the limited samples for which this information was available indicated that findings reported above were unchanged although confidence intervals were wider. For example, women with five or more symptoms had significantly elevated risk for incident MI relative to women with no symptoms when family history was included in the models (multivariable-adjusted OR $=5.89,95 \%$ CI: $1.79-19.40, p<.01$ ), and when BMI was included (multivariable-adjusted OR $=6.94,95 \%$ CI: $2.07-23.33, p<.01$ ). The possibility of reverse causality was considered. Date of event was unavailable for a significant fraction of those with incident MI, but was more often available for cardiac death. However, only two deaths were recorded in the first 2 years, suggesting that reverse causality is unlikely to account for the observed PTSD-CHD associations.

\section{Discussion}

Findings from this study suggest that damaging effects of PTSD symptoms are not limited to military men, but are also evident among civilian women. Community-dwelling women with high levels of PTSD symptoms had over three times the risk of incident CHD relative to women who reported no PTSD symptoms. This effect was maintained after controlling for known coronary risk factors, as well as current depression or trait anxiety, and excluding angina pectoris. Findings are particularly striking given the limited number of CHD events in this sample. It is important to note that past-year PTSD only was assessed. Despite this potentially limited duration of PTSD symptom exposure an increased risk of incident CHD was evident. Because PTSD in this study was assessed only among women who reported exposure to a traumatic event in the past year, women with PTSD resulting from exposure to a traumatic event before the past year would not have been captured in this PTSD assessment; however, such misclassification would likely bias our risk estimates toward the null.

Findings from the present study differ in some important ways from the recently reported findings on PTSD and incident CHD in men. In the prior study, the sample was comprised of men for whom trauma exposure was likely combat-related. In contrast, the present study sample was comprised of civilian, community-dwelling women who were likely exposed to different types of trauma. For example, PTSD in community-dwelling women has been linked with sexual and physical assault, serious accidents or injuries, and natural disasters (Kessler, Sonnega, Bromet, Hughes, \& Nelson, 1995). The ECA sample was also younger compared with the men in the previous study, which likely explains the smaller number of CHD events available for study and the wider confidence intervals around the risk estimates.

Other work has proposed that prolonged stress reactions and high distress lead to impaired adaptation and increased wear and tear on the body which may ultimately initiate disease processes (McEwen, 2003). The time course of PTSD can follow one of several patterns, where 
high symptoms levels after traumatic exposure are followed by recovery, chronic symptoms persist over time, or relapsing — remitting symptoms (Koenen, Stellman, Stellman, \& Sommer, 2003). If PTSD indeed has pathophysiologic or atherogenic effects, they are most likely to be evident when PTSD follows a pattern of persisting or recurring symptoms over time. Specific mechanisms by which PTSD might influence CHD are as yet unknown, but two primary pathways have been proposed. PTSD may directly alter biological processes in ways that lead to atherosclerosis and cardiovascular system damage. PTSD may also motivate health-related behaviors that influence risk of developing CHD. In fact, these are not mutually exclusive processes.

PTSD has been associated with greater likelihood of smoking and excess alcohol consumption, behaviors that increase risk of CHD (Breslau, Davis, \& Schultz, 2003). Consistent with prior findings, in this ECA sample individuals with more PTSD symptoms were also more likely to smoke or consume more than two alcohol drinks per day. Somewhat surprisingly, after controlling for these behaviors and other coronary risk factors, the PTSD-CHD association was stronger. Data on other relevant health-related variables (e.g., exercise frequency, diet) were not available for most participants, and further work is needed to more fully explore whether these behaviors might help to explain the relationship between PTSD and CHD.

Biological alterations that may be attributed to PTSD have been considered. For example, some work has focused on the hyperarousal associated with PTSD and hypothesized that excess cardiovascular reactivity, altered vagal tone, and neuroendocrine dysregulation associated with PTSD may lead to increased CHD risk. Both sympathetic and parasympathetic dysfunctions have been implicated in the pathophysiology of PTSD, with resting states characterized by low vagal tone and excess sympathetic activity (Cohen, Benjamin, Geva, Matar, Kaplan, \& Kotler, 2000). Reduced vagal tone has been identified as a risk factor for sudden cardiac death even among individuals free of diagnosed CHD (Molgaard, Sorensen, \& Bjerregaard, 1991).

Individuals with PTSD also exhibit enhanced negative feedback sensitivity of glucocorticoid receptors in the stress response system, lower than normal urinary and plasma cortisol levels, as well as exaggerated catecholamine responses to trauma-related stimuli (Vanitallie, 2002). PTSD has further been linked with chronically elevated systemic proinflammatory activity and hypercoagulability (von Kanel, Hepp, Buddeberg, et al., 2006; von Kanel, Hepp, Kraemer, et al., 2006). Together these processes may cause or exacerbate endothelial damage and promote the development of atherosclerosis (Schneiderman, 1987).

Findings from this study have several limitations. It is possible there was a differential rate of CHD events among the 285 women who were lost to follow-up at Wave 3, and if they were included in our sample, findings would change. Only a small number of CHD events occurred in this sample limiting the analyses and their interpretation. Relatedly, whether PTSD is associated with CHD among community dwelling men is also of interest; however, levels of PTSD symptoms among men in this sample were quite low making it difficult to examine this question. Neither exposure to trauma nor the nature of the trauma was specifically evaluated, and we lacked or had incomplete information on several traditional risk factors, including cholesterol, BMI, and family history of heart disease. Moreover, assessment of diabetes may have been somewhat underreported as criteria have changed since the Wave 2 assessment. Evaluation of lifetime occurrence of PTSD was not available, but may be expected to increase the magnitude of the observed effects. The CHD measure is based on self-report and may be subject to misclassification, and does not include all possible cardiac outcomes (e.g., congestive heart failure, arrhythmias). Other work has considered the level of agreement between selfreported $\mathrm{MI}$ and medical records, and reported agreement rates ranging from $53 \%$ to $81 \%$, but also noted that misclassification is most commonly with other cardiovascular diagnoses (O’Donnell et al., 1999). Moreover, although there is evidence that distressed individuals with PTSD are more likely to report more illness-related symptoms, as well as be high utilizers in 
the health care system (de Waal, Arnold, Spinhoven, Eekhof, \& van Hemert, 2005; Gerber et al., 1992; Kisely, Goldberg, \& Simon, 1997; Kroenke, Jackson, \& Chamberlin, 1997; Watson $\&$ Pennebaker, 1989), there is little evidence that suggests distressed individuals are more likely to report a diagnosed illness in the absence of ever receiving such a diagnosis. The prospective nature of the data collection with PTSD assessment based on clinical criteria and occurring well before ascertainment of coronary outcomes, and the consistency of our findings with other work using objectively measured outcomes strengthens confidence in our findings and decreases the possibility of recall or information bias.

This is the first prospective study of PTSD and CHD in civilian women. The strength of the association after controlling for known risk factors and depression or trait anxiety, and its consistency with recently reported findings among men suggests that the relationship is valid and may operate in diverse groups. PTSD has been identified as a marker of extreme distress in response to a potentially traumatic event. These data suggest that the prolonged stress that can occur in response to trauma exposure not only increases risk for serious mental health problems, but may also increase risk of CHD. The duration or chronicity of PTSD that is necessary to initiate pathophysiological processes is unclear. Moreover, it is as yet unknown how reversible these pathophysiologic processes are and whether effective treatment for PTSD will also reduce coronary risk. However, from a public health perspective, it may be prudent to view individuals with PTSD as an at-risk population, and implement preventive and intervention strategies accordingly.

\section{Acknowledgments}

Koenen is supported by NIMH Grants K08-MH070627 and MH078928. Eaton is supported by NIMH 47447 and Jones is supported by the T32 MH 14592.

\section{References}

Badawi MA, Eaton WW, Myllyluoma J, Weimer LG, Gallo J. Psychopathology and attrition in the Baltimore ECA 15-year follow-up 1981-1996. Social Psychiatry and Psychiatric Epidemiology 1999;34:91-98. [PubMed: 10189815]

Breslau N, Davis GC, Schultz LR. Posttraumatic stress disorder and the incidence of nicotine, alcohol, and other drug disorders in persons who have experienced trauma. Archives of General Psychiatry 2003;60:289-294. [PubMed: 12622662]

Cohen H, Benjamin J, Geva AB, Matar MA, Kaplan Z, Kotler M. Autonomic dysregulation in panic disorder and in post-traumatic stress disorder: Application of power spectrum analysis of heart rate variability at rest and in response to recollection of trauma or panic attacks. Psychiatry Research 2000;96:1-13. [PubMed: 10980322]

de Waal MW, Arnold IA, Spinhoven P, Eekhof JA, van Hemert AM. The reporting of specific physical symptoms for mental distress in general practice. Journal of Psychosomatic Research 2005;59:89-95. [PubMed: 16186004]

Dong M, Giles WH, Felitti VG, Dube SR, Williams JE, Chapman DP, et al. Insights into causal pathways for ischemic heart disease: Adverse childhood experiences study. Circulation 2004;110:1761-1766. [PubMed: 15381652]

Gerber PD, Barrett JE, Barrett JA, Oxman TE, Manheimer E, Smith R, et al. The relationship of presenting physical complaints to depressive symptoms in primary care patients. Journal of General Internal Medicine 1992;7:170-173. [PubMed: 1487765]

Hoge CW, Castro CA, Messer SC, McGurk D, Cotting DI, Koffman RL. Combat duty in Iraq and Afghanistan, mental health problems, and barriers to care. New England Journal of Medicine 2004;351:13-22. [PubMed: 15229303]

Kang HK, Bullman TA, Taylor JW. Risk of selected cardiovascular diseases and posttraumatic stress disorder among former World War II prisoners of war. Annals of Epidemiology 2006;16:381-386. [PubMed: 15994096] 
Kessler RC, Berglund P, Demler O, Jin R, Merikangas KR, Walters EE. Lifetime prevalence and ageof-onset distributions of $D S M-I V$ disorders in the National Comorbidity Survey Replication. Archives of General Psychiatry 2005;62:593-602. [PubMed: 15939837]

Kessler RC, Sonnega A, Bromet E, Hughes M, Nelson CB. Posttraumatic stress disorder in the National Comorbidity Survey. Archives of General Psychiatry 1995;52:1048-1060. [PubMed: 7492257]

Kisely S, Goldberg D, Simon G. A comparison between somatic symptoms with and without clear organic cause: Results of an international study. Psychological Medicine 1997;27:1011-1019. [PubMed: 9300507]

Koenen KC, Stellman JM, Stellman SD, Sommer JF. Risk factors for course of posttraumatic stress disorder among Vietnam veterans: A 14-year follow-up of American Legionnaires. Journal of Consulting and Clinical Psychology 2003;71:980-986. [PubMed: 14622073]

Kroenke K, Jackson JL, Chamberlin J. Depressive and anxiety disorders in patients presenting with physical complaints: Clinical predictors and outcome. American Journal of Medicine 1997;103:339_ 347. [PubMed: 9375700]

Kubzansky LD, Kawachi I. Going to the heart of the matter: Do negative emotions cause coronary heart disease? Journal of Psychosomatic Research 2000;48:323-337. [PubMed: 10880655]

Kubzansky LD, Koenen KC. Is post-traumatic stress disorder related to development of heart disease? Future, Cardiology 2007;3:153-156.

Kubzansky LD, Koenen KC, Spiro A, Vokonas PS, Sparrow D. Prospective study of post-traumatic stress disorder symptoms and coronary heart disease in the Normative Aging Study. Archives of General Psychiatry 2007;64:109-116. [PubMed: 17199060]

McEwen BS. Mood disorders and allostatic load. Biological Psychiatry 2003;54:200-207. [PubMed: 12893096]

Molgaard H, Sorensen KE, Bjerregaard P. Attenuated 24-h heart rate variability in apparently healthy subjects, subsequently suffering sudden cardiac death. Clinical Autonomic Research 1991;1:223233.

O’Donnell CJ, Glynn RJ, Field TS, Averback R, Satterfield S, Friesenger GC 2nd, et al. Misclassification and under-reporting of acute myocardial infarction by elderly persons: Implications for communitybased observational studies and clinical trials. Journal of Clinical Epidemiology 1999;52:745-751. [PubMed: 10465319]

Pratt LA, Ford DE, Crum RM, Armenian HK, Gallo JJ, Eaton WW. Depression, psychotropic medication, and risk of myocardial infarction: Prospective data from the Baltimore ECA follow-up. Circulation 1996;94:3123-3129. [PubMed: 8989119]

Regier DA, Myers JK, Kramer M, Robins LN, Blazer DG, Hough RL, et al. The NIMH Epidemiologic Catchment Area program. Historical context, major objectives, and study population characteristics. Archives of General Psychiatry 1984;41:934-941. [PubMed: 6089692]

Robins LN, Helzer JE, Croughan J, Ratcliff KS. National Institute of Mental Health Diagnostic Interview Schedule: Its history, characteristics, and validity. Archives of General Psychiatry 1981;38:393-398. [PubMed: 7212969]

Rosamond WD, Flegal K, Friday G, Furie K, Go A, Greenlund K, et al. Heart Disease and Stroke Statistics-2007 Update: A report from the American Heart Association Statistics Committee and Stroke Statistics Subcommittee. Circulation 2007;115:e70-e171.

Rozanski A, Blumenthal JA, Davidson KW, Saab P, Kubzansky LD. The epidemiology, pathophysiology, and management of psychosocial risk factors in cardiac practice: The emerging field of behavioral cardiology. Journal of American College of Cardiology 2005;45:637-651.

SAS Institute. SAS/STAT User's Guide (Version 6). Vol. fourth. Cary, NC: SAS Institute Inc; 1990.

Schneiderman N. Psychophysiologic factors in atherogenesis and coronary artery disease. Circulation 1987;76(Pt 2):I41-47. [PubMed: 3297408]

Spindler H, Pedersen SS. Posttraumatic stress disorder in the wake of heart disease: Prevalence, risk factors, and future research directions. Psychosomatic Medicine 2005;67:715-723. [PubMed: 16204429]

Vanitallie TB. Stress: A risk factor for serious illness. Metabolism: Clinical and Experimental 2002;51 (Suppl 1):40-45. [PubMed: 12040540] 
von Kanel R, Hepp U, Buddeberg C, Keel M, Mica L, Aschbacher K, et al. Altered blood coagulation in patients with posttraumatic stress disorder. Psychosomatic Medicine 2006;68:598-604. [PubMed: 16868270]

von Kanel R, Hepp U, Kraemer B, Traber R, Keel M, Mica L, et al. Evidence for low-grade systemic proinflammatory activity in patients with posttraumatic stress disorder. Journal of Psychiatric Research 2006;41:744-752. [PubMed: 16901505]

Watson D, Pennebaker JW. Health complaints, stress, and distress: Exploring the central role of negative affectivity. Psychological Review 1989;96:234-254. [PubMed: 2710874] 
Table 1

Distribution of Coronary Risk Factors in Women in the Baltimore Epidemiologic Catchment Area Study

\begin{tabular}{|c|c|c|c|}
\hline \multirow[b]{2}{*}{ Risk factor } & \multicolumn{3}{|c|}{ PTSD symptoms } \\
\hline & $\mathbf{0}$ & $1-4$ & $5+$ \\
\hline Number in sample & $934(89.1 \%)$ & $74(7.0 \%)$ & $42(4.0 \%)$ \\
\hline Age at baseline (years) $(p=.0800)$ & 45.8 & 40.3 & 44.2 \\
\hline \multicolumn{4}{|l|}{ Race/ethnicity } \\
\hline White & $558(59.2 \%)$ & $51(68.9 \%)$ & $25(59.5 \%)$ \\
\hline Non-White & $385(40.8 \%)$ & $23(31.2 \%)$ & $17(40.5 \%)$ \\
\hline Current smokers (\%) & $402(42.7 \%)$ & $31(41.9 \%)$ & $24(57.1 \%)$ \\
\hline Former smokers $(\%)$ & $232(24.7 \%)$ & $15(20.3 \%)$ & $9(21.4 \%)$ \\
\hline History of high blood pressure & $316(33.7 \%)$ & $20(27.0 \%)$ & $15(35.7 \%)$ \\
\hline History of diabetes & $70(7.5 \%)$ & $3(4.1 \%)$ & $1(2.4 \%)$ \\
\hline $\begin{array}{l}\text { Consume two or more drinks of alcohol per } \\
\text { day } a\end{array}$ & $158(16.8 \%)$ & $17(23.0 \%)$ & $8(19.1 \%)$ \\
\hline Educational attainment high school or more & $484(51.3 \%)$ & $46(62.2 \%)$ & $21(50.0 \%)$ \\
\hline \multicolumn{4}{|l|}{ Income $(p<.0001)$} \\
\hline$<\$ 14,999$ & $467(55.8 \%)$ & $31(47.0 \%)$ & $24(61.5 \%)$ \\
\hline$\$ 15,000-24,000$ & $202(24.1 \%)$ & $16(24.2 \%)$ & $6(15.4 \%)$ \\
\hline$\$ 25,000$ & $168(20.1 \%)$ & $19(28.8 \%)$ & $9(23.1 \%)$ \\
\hline Current depression $(p<.001)$ & $12(1.3 \%)$ & $1(1.4 \%)$ & $5(11.9 \%)$ \\
\hline Trait anxiety $(p<.0001)$ & $189(20.1 \%)$ & $29(39.2 \%)$ & $22(52.4 \%)$ \\
\hline
\end{tabular}

${ }^{a}$ Reported values are $N(\%)$. 
Table 2

Age-Adjusted and Multivariable Odds Ratio (OR) of CHD According to Level of PTSD Symptoms (Confidence Intervals are in Parentheses) in Women in the Baltimore Epidemiologic Catchment Area Study

\begin{tabular}{|c|c|c|c|}
\hline & \multicolumn{3}{|c|}{ Level of PTSD symptoms } \\
\hline & $\mathbf{0}$ & $1-4$ & $5+$ \\
\hline \multicolumn{4}{|l|}{ Total cardiac outcomes ${ }^{\dagger}$} \\
\hline Cases ${ }^{t}$ & 74 & 5 & 7 \\
\hline Age-adjusted OR & 1.00 & $1.22(0.46-3.24)$ & $3.21 *(1.29-7.98)$ \\
\hline Multivariable-adjusted $\mathrm{OR}^{\S}$ & 1.00 & $0.73(0.21-2.53)$ & $3.46^{* *}(1.35-8.90)$ \\
\hline \multicolumn{4}{|l|}{ Total MI } \\
\hline Cases ${ }^{t}$ & 48 & 4 & 5 \\
\hline Age-adjusted OR & 1.00 & $1.50(0.51-4.42)$ & $3.25^{*}(1.16-9.11)$ \\
\hline Multivariable-adjusted $\mathrm{OR}^{\S}$ & 1.00 & $1.24(0.35-4.34)$ & $3.36^{*}(1.15-9.76)$ \\
\hline \multicolumn{4}{|l|}{ Noncardiac mortality } \\
\hline Cases ${ }^{*}$ & 190 & 9 & 7 \\
\hline Age-adjusted OR & 1.00 & $0.77(0.35-1.72)$ & $1.00(0.39-2.56)$ \\
\hline Multivariable-adjusted $\mathrm{OR}^{\S}$ & 1.00 & $0.99(0.41-2.41)$ & $0.85(0.31-2.34)$ \\
\hline \multicolumn{4}{|l|}{$* x<.05$} \\
\hline \multicolumn{4}{|c|}{${ }^{\dagger}$ Angina, nonfatal MI, fatal CHD, and other cardiac death combined. } \\
\hline
\end{tabular}

